\title{
Progression Potential of Ductal Carcinoma in situ Assessed by Genomic Copy Number Profiling
}

\author{
Mina Kitamura ${ }^{a}$ b Takahisa Nakayama $^{a}$ Ken-ichi Mukaisho ${ }^{a}$ Tsuyoshi Mori $^{b}$ \\ Tomoko Umeda $^{b}$ Suzuko Moritanic Ryoji Kushimac Masaji Tani ${ }^{\mathrm{b}}$ \\ Hiroyuki Sugihara ${ }^{a}$ \\ a Division of Molecular Diagnostic Pathology, Department of Pathology, Shiga University of Medical Science,

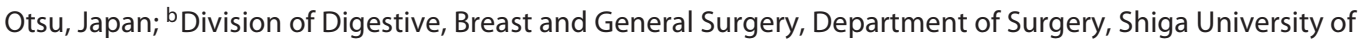 \\ Medical Science, Otsu, Japan; ' Division of Diagnostic Pathology, Shiga University of Medical Science Hospital, \\ Otsu, Japan
}

\section{Keywords}

Copy number alterations - Array-based comparative genomic hybridization - Breast cancer - Ductal carcinoma in situ $\cdot$ Unsupervised hierarchical clustering

\begin{abstract}
Background: Ductal carcinoma in situ (DCIS) of the breast is heterogeneous in terms of the risk of progression to invasive ductal carcinoma (IDC). To treat DCIS appropriately for its progression risk, we classified individual DCIS by its profile of genomic changes into 2 groups and correlated them with clinicopathological progression factors. Methods: We used surgically resected, formalin-fixed, paraffin-embedded tissues of 22 DCIS and 30 IDC lesions. We performed immunohistochemical intrinsic subtyping, array-based comparative genomic hybridization, and unsupervised clustering. Results: The samples were divided into 2 major clusters, $A$ and B. Cluster A showed a greater number of gene and chromosome copy number alterations, a larger IDC/DCIS ratio, a higher frequency of nonluminal subtype, a lower frequency of luminal subtype, and a higher nuclear grade, when com-
\end{abstract}

\section{KARGER}

() 2018 S. Karger AG, Basel

E-Mail karger@karger.com

www.karger.com/pat pared with cluster $B$. However, there was no difference in the frequencies of lymph node metastasis between clusters $A$ and $B$. We identified 9 breast-cancer-related genes, including TP53 and GATA3, that highly contributed to the discrimination of $A$ and $B$ clusters. Conclusion: Classification of breast tumors into rapidly progressive cluster $A$ and the other (cluster B) may contribute to select the treatment appropriate for their progression risk.

(c) 2018 S. Karger AG, Basel

\section{Introduction}

Ductal carcinoma in situ (DCIS) is characterized by noninvasive spreading within mammary ducts. Whether DCIS inevitably becomes invasive and what factors determine the rate of progression remains unclear.

In 2011, breast cancer was newly detected in approximately 48,000 Japanese women, including $14.2 \%$ with DCIS, whereas the percentage of DCIS was only $8.2 \%$ in 2004. This increase in the proportion of DCIS may reflect an increase in the number of breast cancer patients who 
were detected by mammographic screening [1]. Mammographic screening is more prevalent in North America than in Japan, and the number of DCIS cases detected by screening accounts for nearly $20 \%$ of all breast cancers in recent years [2]. This increase in DCIS detection is likely to continue until it reaches the prevalence of latent DCIS, which is as high as $8.9 \%$ of autopsy cases [3].

If DCIS inevitably becomes invasive ductal carcinoma (IDC), the screening-based detection of DCIS can lead to a reduction in the mortality of breast cancer patients. Many studies reported that screenings had actually reduced breast cancer mortality but that the reduction size was smaller than expected probably because of overdiagnosis of very dormant tumors as cancers $[4,5]$. A retrospective cohort study demonstrated that there was no significant difference in the weighted hazard ratios of breast cancer-specific 10-year survival between surgery and nonsurgery groups for low-grade DCIS [6]. Accordingly, a follow-up study for up to 20 years after a biopsy diagnosis of DCIS without subsequent treatment reported that $28 \%$ of these patients recurred for IDC within approximately 15 years [7]. This study and other similar reports $[3,8]$ suggest that some DCIS lesions remain dormant and have a very slow progression rate to IDC. It is also possible that some DCIS lesions do not become invasive.

Molecular and epidemiological data indicate that breast cancer development is a multi-step process [9]. The progression from DCIS to IDC may involve stepwise genetic alterations $[10,11]$. Some studies have suggested that copy number aberrations (CNAs) are associated with the progression from DCIS to IDC, including amplifications of MYC [12], FGFR1 [13], and CCND1 [14], which were more frequently observed in IDC than DCIS. However, other studies comparing the DCIS and invasive components from the same patient demonstrated that CNAs at the chromosome level were very similar between intraductal and invasive components [15]. Analogous approaches searching for common transcriptomic and/ or genomic differences between matched synchronous DCIS and IDC have been unsuccessful [16, 17]. Current studies have also failed to identify driver genes that play a significant role in the transition from DCIS to IDC [2]. Thus, the presence of progression-related genomic changes and overall genomic similarity between DCIS and IDS requires further study.

In the present study, we focused on the CNA profiles of genomic DNA using array-based comparative genomic hybridization (CGH). These profiles are unique for individual neoplasms because they include random gene alterations that have a neutral role for carcinogenesis, accumulate over time based on genetic instability, and are selected by the tissue microenvironment. Approximately three quarters of the natural history of solid cancers has elapsed once tumors reach $1 \mathrm{~cm}$ in diameter [18], which leaves a limited opportunity for the accumulation of additional genomic changes. Thus, the CNA profile of clinically detectable breast cancer may already include information enough for outcome prediction. In our previous gastric cancer studies, we classified the samples based on their CNA profiles using unsupervised hierarchical cluster analyses and demonstrated that nearly all early cancer of the undifferentiated type can become advanced [19], whereas approximately $80 \%$ of noninvasive neoplasms of the differentiated type showed a lineage distinct from advanced cancers [20].

Applying the similar approach to breast cancers, in the present study, we have found that breast tumors, including DCIS, IDC and papillomas, were classified into a rapidly progressive group and slowly progressive group. This classification may contribute to select the treatment of individual breast tumors appropriate for their progression risk.

\section{Methods}

\section{Patients}

The study consisted of 50 patients who underwent a partial or total mastectomy for DCIS lesions $(n=22)$ or IDCs $(n=30 ; 15 \mathrm{~T} 1$, 14 T2, and 1 T4 tumors) from December 2009 to January 2014 (online suppl. file 1; for all online suppl. material, see www.karger. com/doi/10.1159/000492833). Two patients had 2 tumors: 1 patient (No. 13) with bilateral IDC and DCIS and 1 patient (No. 17) with unilateral IDC and DCIS in areas A and C, respectively. All patients were female. The mean age was 55.2 years (range, 35-84 years). None of the patients received any preoperative radio- and/ or chemotherapy. The conduct in this study was approved by the Institutional Review Board at the Shiga University of Medical Science on the condition that the materials used remained anonymous (Permission No.: 26-36 on July 24, 2014). Written informed consent was not required in this retrospective study because of the use of archival materials and detected acquired genomic copy number changes.

Tissue Samples

We used formalin-fixed, paraffin-embedded tissues. Tissues were fixed in buffered $10 \%$ formalin for $24-48 \mathrm{~h}$. In 20 of the 30 IDCs, DNA samples were taken from both ductal and invasive components. DNA samples of metastatic tumors in lymph nodes were available in 7 cases (online suppl. file 1). Only patient No. 18 had distant metastasis, from which no sample was available. N2 or $\mathrm{N} 3$ nodal metastasis was not detected in any of the patients in this study. 
Immunohistochemistry and in situ Hybridization

We used 3- $\mu \mathrm{m}$-thick tissue sections for the immunohistochemical (IHC) analysis of the estrogen receptor (ER), progesterone receptor, HER2, basal and myoepithelial markers (cytokeratin 5/6 and p63), and Ki-67. We used the following antibodies: anti-ER (clone 1D5, DAKO, Santa Clara, CA, USA; dilution 1:50), antiprogesterone receptor (clone PgR636, DAKO; dilution 1:50), antiKi-67 (clone MM1, Leica Biosystems Newcastle Ltd., Newcastle upon Tyne, UK; dilution 1:100), anti-HER2 (clone 4B5, Ventana, Tucson, AZ, USA; prediluted), and anti-cytokeratin 5/6 and antip63 (based on a previous study) [21]. IHC analysis was optimized by evaluating serial sections with multiple antibody concentrations using a Discovery Automated Immunostainer (Ventana Medical Systems, Tucson, AZ, USA).

For HER2 testing, dual-color fluorescence in situ hybridization (FISH), using the PathVysion HER-2 DNA Probe Kit (PathVysion; Abbott Molecular, Des Plaines, IL, USA), and IHC assessment were used in accordance with the guidelines of the American Society of Clinical Oncology (ASCO) [22]. After counting the signals of HER2 and those of centromeric enumeration probe 17 (CEP17) under a fluorescence microscope, a HER2/CEP17 ratio $\geq 2.0$ was defined as amplification.

\section{Genomic DNA Extraction}

Tumor and normal lymph node (reference) samples were obtained from 5 - $\mu \mathrm{m}$-thick tissue sections using a laser microdissection system (LMD6000; Leica Microsystems, Wetzlar, Germany) [20]. Briefly, each sample was dissected from an area $\geq 6 \mathrm{~mm}^{2}$. In tumor samples, neoplastic cells comprised $90 \%$ of the total cell count. The cells were digested with a $200 \mathrm{mg} / \mathrm{mL}$ proteinase K solution (P2308, Sigma-Aldrich, St. Louis, MO, USA) for $70 \pm 2 \mathrm{~h}$ at $37^{\circ} \mathrm{C}$ prior to a phenol/chloroform DNA extraction. DNA quality was assessed based on the A260/A280 ratio (cutoff $>1.5$ ), A260/ $\mathrm{A} 230$ ratio (cutoff $>1.0$ ), and the presence or absence of doublestranded DNA.

\section{Whole Genome Amplification}

Sample DNA was amplified using the GenomePlex Whole Genome Amplification Kit (WGA2 Kit; Sigma, St. Louis, MO, USA) according to the manufacturer's protocol [23].

\section{Array CGH}

For genomic DNA analysis, a 60-mer oligonucleotide CGH microarray (Agilent, Santa Clara, CA, USA) was used according to the manufacturer's instructions [24]. The genomic DNA enzymatic labeling and subsequent array CGH were performed as previously described [20]. The tumor-to-reference fluorescence intensity ratio $(\mathrm{T} / \mathrm{R})$ was calculated from the hybridized array images obtained. The UCSC Genome Browser was used with the latest resource content: hg19 assembly - Design ID 021429 (GRCh Build 37). CNAs were defined as a gain, loss, and amplification when the base 2 logarithm of the T/R ratio was $>0.3219$, $<-0.3219$, and $>1.0$, respectively. The microarray data were registered in the Gene Expression Omnibus database (Accession No.: GSE86988).

\section{Validation of Array CGH Data by FISH}

Using the samples positive for ERBB2 gene amplification, we compared FISH signal numbers and the $\mathrm{T} / \mathrm{R}$ ratio of the array CGH. For dual-color FISH for ERBB2 gene amplification, we used a PathVysion HER2 DNA Probe Kit (Abbot Molecular Inc.). We randomly selected 7 and 8 samples from the sample groups that showed strong $(3+)$ and weak $(1+$ or $2+)$ HER2 immunoreactivities, respectively.

\section{Clustering Algorithm}

To enhance the signal-to-noise ratio in the hybridization analysis, we averaged the $T / R$ ratio of the probes within each gene prior to performing the cluster analyses. The noise-canceling effect of averaging depends on the gene size (probe number within the gene), whereas the clustering reproducibility becomes lower as the gene number becomes smaller. Notably, larger gene sizes correspond to smaller gene numbers. Thus, we repeated the clustering analysis to determine the optimal gene size and number.

To classify samples based solely on genome-wide similarities in gene copy number gain/loss patterns, we performed an unsupervised hierarchical cluster analysis using a free software program (Cluster 3.0, version 1.52, and TreeView, version 1.1.6r2). The clustering results were assessed as previously described [20, 25, 26]. Briefly, we repeated the clustering analysis using genes ranging from 370 genes containing $\geq 10$ probes to 9,487 genes containing $\geq 2$ probes. We selected 2 gene size conditions that showed the highest reproducibility in clustering dendrograms, and then selected the condition with the highest proportion of sample sets that derived from the same tumor and showed the neighboring in the clustering dendrogram.

The clustering condition was set to a complete linkage (maximum of distance metric on similarities) and the uncentered correlation distance (distance measures based on modified Pearson's correlation).

\section{Statistical Analysis}

The CNA differences for each gene between clusters A and B were statistically assessed in an unequal sample size $t$ test (Welch's $t$ test). A bilateral $p$ value of $\leq 0.05$ was considered statistically significant. For multiple comparisons, the $t$ test was subsequently adjusted using the Bonferroni correction [25] (Microsoft Office Excel 2013). To assess trend differences in either nuclear atypia or CNA accumulations between the 2 groups, a Fisher's exact test $(2 \times 2$ contingency tables) was performed (BellCurve for Excel, Social Survey Research Information Co. Ltd., Tokyo, Japan).

\section{Results}

\section{Immunohistochemistry}

The intrinsic subtype was estimated immunohistochemically on the basis of the clinicopathological surrogate definitions of subtypes based on the 2013 St. Gallen Consensus [27]; immunohistochemistry-based definition of luminal A-like tumors is ER-positive, HER2-negative, Ki-67 index less than 14\%, and PR positivity more than $20 \%$ [28]. The 81 carcinoma samples were classified into 20 luminal A-like, 37 luminal B-like (HER2 negative), 6 luminal B-like (HER2 positive), 6 HER2 positive (nonluminal), and 12 triple negative (ductal). 


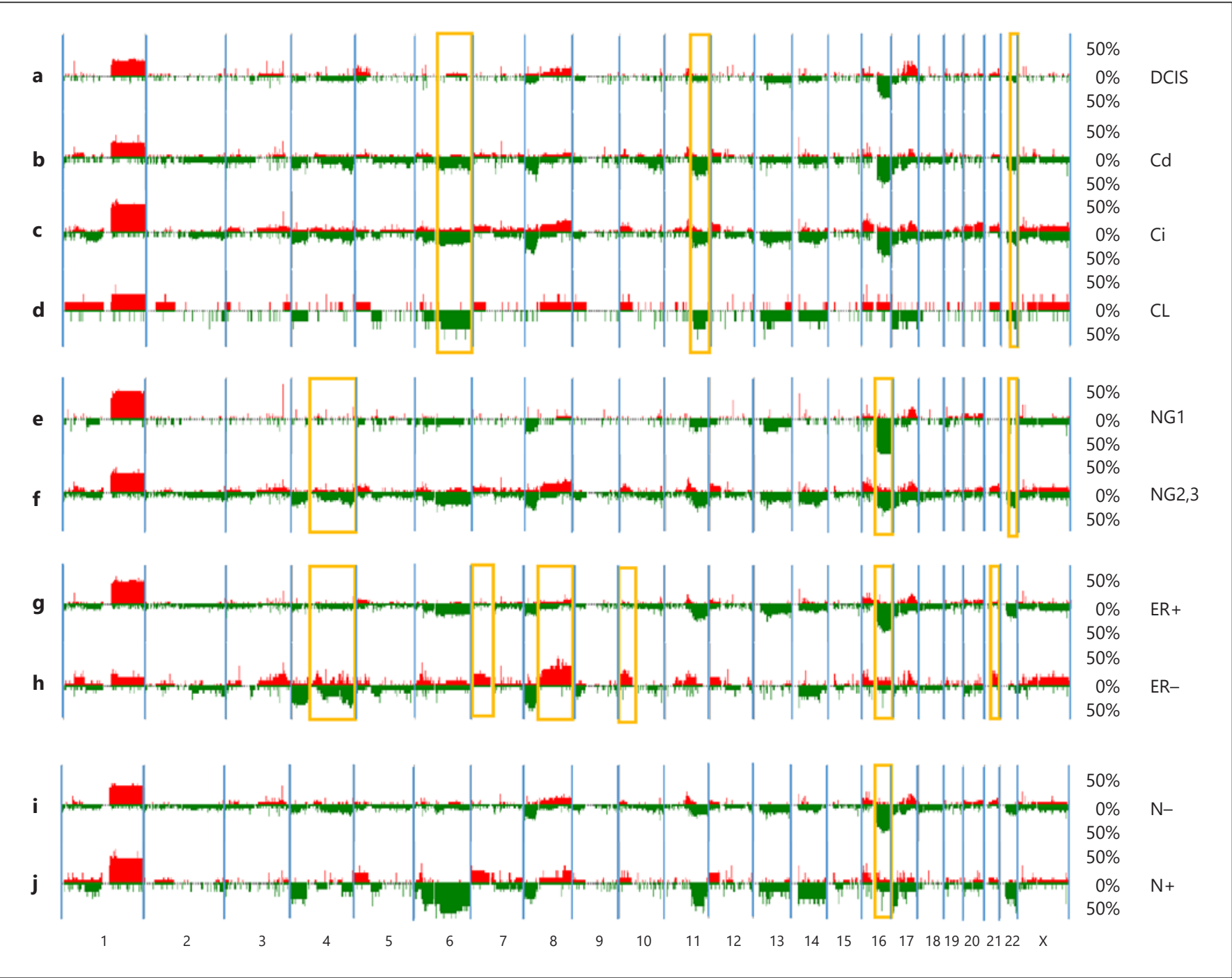

Fig. 1. Penetrance plots. a Ductal carcinoma in situ (DCIS, 24 samples). b Ductal part of invasive ductal carcinoma (IDC) (Cd, 22 samples). c Invasive part of IDC (Ci, 28 samples). d Nodal metastatic tumors of IDC (CL, 7 samples). e Low nuclear grade (NG1, 22 samples). f High nuclear grade (NG2, 3, 59 samples). g Luminal subtype (ER+, 63 samples). h Nonluminal subtype (ER-, 18 samples). i Nodal metastasis absent ( $\mathrm{N}-, 37$ samples). j Nodal metastasis present $(\mathrm{N}+, 20$ samples). Yellow squares mark statistically significant changes.

2). Only $16 q-$ was significantly different in frequency between the samples of N0 and N1 tumors (Fig. 1i, j, Table 3).

Amplifications of $17 \mathrm{q} 12$ were found in $9 / 12$ of the tumor samples that showed strong (3+) IHC expression of HER2 irrespective of DCIS or IDC.

\section{Validation of Array CGH Data by FISH}

In the 7 samples with strong HER2 immunoreactivity, the $\mathrm{T} / \mathrm{R}$ ratios of the $E R B B 2$ gene were 1.81-3.12 (average 2.53 ), and the FISH signal ratio was 2.4-5.8 (average 4.2). 
In the 8 samples with weak HER2 immunoreactivity, the $\mathrm{T} / \mathrm{R}$ ratios of $E R B B 2$ gene were -0.93 to 0.03 (average -0.24 ), and the FISH signal ratio was 0.9-1.3 (average 1.05). No gene amplification is detected in the samples with weak HER2 immunoreactivity, and vice versa.

Table 1. Comparisons of clinicopathological features and chromosomal changes between DCIS and IDC samples

\begin{tabular}{llll}
\hline & DCIS & IDC & p value \\
\hline Low NG (1) & 10 & 12 & 0.0986 \\
High NG $(2,3)$ & 14 & 45 & \\
ER+ (luminal A/B; HER2+/-) & 20 & 43 & 0.5637 \\
ER- (HER2+/TN) & 4 & 14 & \\
6q- & 0 & 10 & 0.0290 \\
$\quad$ Absent & 24 & 47 & \\
8p- & 2 & 13 & 0.2094 \\
$\quad$ Absent & 22 & 44 & \\
$11 \mathrm{q}-$ & 1 & 16 & 0.0167 \\
$\quad$ Absent & 23 & 41 & \\
16p+ & 2 & 10 & 0.4943 \\
$\quad$ Absent & 22 & 47 & \\
20q+ & 1 & 5 & 0.6641 \\
$\quad$ Absent & 23 & 52 & \\
22q- & 1 & 15 & 0.0300 \\
$\quad$ Absent & 23 & 40 & \\
\hline
\end{tabular}

NG, nuclear grade; TN, triple negative.

\section{Clustering of Gene Copy Number Profiles}

The individual probe $T / R$ ratios within a specified gene were averaged. The average T/R ratios of 30,471 gene regions were calculated from 55,023 probes. Genes selected based on size were subjected to an unsupervised hierarchical cluster analysis. After repeated clustering using varying minimum gene sizes, we found that the gene sizes of $\geq 3$ probes and $\geq 4$ probes gave highest reproducibility of clustering results (online suppl. file 2 ). In the condition of a gene size $\geq 4$ probes, all samples from the same case were in the neighboring position in the clustering dendrogram, confirming the reproducibility of the CNA profile. Thus, we adopted this as the optimal condition for the unsupervised hierarchical clustering of all (cancer and papilloma) samples. This condition yielded 2 main clusters: A and B (Fig. 2). The cancer samples of cluster A showed a greater IDC/DCIS ratio, a higher ER-/ER+ (nonluminal/luminal) ratio, and higher nuclear grade (NG) than those in cluster B (Table 4). There were also tendencies for a higher Ki-67 index, higher triple negative tumors in cluster A. There was no difference in the frequency of lymph node metastasis between clusters A and B.

\section{Lineage-Specific Chromosomal CNA Profile}

The penetrance plots of clusters A and B revealed distinct chromosomal CNA profiles (Fig. 3). In addition to changes common to these clusters (a gain of $1 \mathrm{q}$ and stage-

Table 2. Differences in sample-based frequencies of chromosomal copy number changes between high and low nuclear grade (NG), between $\mathrm{ER}+$ and $\mathrm{ER}-$, and between clusters A and B

\begin{tabular}{|c|c|c|c|c|c|c|c|c|c|}
\hline & \multicolumn{3}{|c|}{ Nuclear grades } & \multicolumn{3}{|c|}{ Estrogen receptors } & \multicolumn{3}{|l|}{ Clusters } \\
\hline & \multicolumn{2}{|c|}{ number of samples } & \multirow{2}{*}{$p$ value } & \multicolumn{2}{|c|}{ number of samples } & \multirow{2}{*}{$p$ value } & \multicolumn{2}{|c|}{ number of samples } & \multirow{2}{*}{$p$ value } \\
\hline & $\begin{array}{l}\text { high NG } \\
(n=59)\end{array}$ & $\begin{array}{l}\text { low NG } \\
(n=22)\end{array}$ & & $\begin{array}{l}\mathrm{ER}+ \\
(n=63)\end{array}$ & $\begin{array}{l}\mathrm{ER}- \\
(n=18)\end{array}$ & & $\begin{array}{l}\text { cluster A } \\
(n=50)\end{array}$ & $\begin{array}{l}\text { cluster B } \\
(n=31)\end{array}$ & \\
\hline $1 q+$ & 21 & 9 & 0.7966 & 25 & 3 & 0.0938 & 17 & 11 & 1.0000 \\
\hline $4 p-$ & 10 & 1 & 0.2731 & 5 & 6 & 0.0110 & 11 & 0 & 0.0055 \\
\hline $4 q-$ & 13 & 0 & 0.0157 & 6 & 7 & 0.0067 & 10 & 3 & 0.3511 \\
\hline $5 p+$ & 8 & 1 & 0.432 & 6 & 3 & 0.4083 & 9 & 0 & 0.0112 \\
\hline $7 \mathrm{p}+$ & 6 & 0 & 0.1824 & 2 & 4 & 0.0201 & 6 & 0 & 0.0774 \\
\hline $8 p-$ & 12 & 3 & 0.7486 & 9 & 6 & 0.3399 & 15 & 0 & 0.0003 \\
\hline $8 q+$ & 13 & 1 & 0.0975 & 5 & 9 & 0.0201 & 11 & 3 & 0.1466 \\
\hline $10 \mathrm{p}+$ & 6 & 0 & 0.1824 & 1 & 5 & 0.0017 & 6 & 0 & 0.0783 \\
\hline $13 \mathrm{q}-$ & 7 & 3 & 1.0000 & 10 & 0 & 0.1067 & 6 & 4 & 1.0000 \\
\hline $14 \mathrm{q}^{-}$ & 8 & 0 & 0.0999 & 6 & 2 & 1.0000 & 7 & 1 & 0.1453 \\
\hline $16 \mathrm{p}+$ & 11 & 1 & 0.1648 & 7 & 5 & 0.1262 & 11 & 1 & 0.0267 \\
\hline $16 \mathrm{q}-$ & 14 & 12 & 0.0148 & 25 & 1 & 0.0083 & 13 & 13 & 0.1501 \\
\hline $17 \mathrm{p}-$ & 13 & 1 & 0.0975 & 11 & 3 & 1.0000 & 12 & 2 & 0.0675 \\
\hline $21 \mathrm{q}+$ & 7 & 0 & 0.1809 & 2 & 5 & 0.0052 & 7 & 0 & 0.0401 \\
\hline $22 \mathrm{q}-$ & 16 & 0 & 0.0042 & 14 & 2 & 0.5027 & 10 & 6 & 1.0000 \\
\hline
\end{tabular}


specific losses of $6 \mathrm{q}$ and $16 \mathrm{q}$ ), cluster A was characterized by gains of $5 p, 16 p$, and $21 q$ and losses of $4 p$ and $8 p$, whereas cluster B scarcely showed these changes (Table 2 ).

\section{Genes Exhibiting Significantly Different CNAs}

\section{between 2 Major Clusters}

We extracted 728 genes that show significantly different mean copy numbers between clusters $\mathrm{A}$ and $\mathrm{B}$ in a $t$ test with Bonferroni correction. Out of the top 45 genes shown in online supplementary file $3 \mathrm{a}, 42$ were proteincoding, including 10 genes that showed concordance between the gene CNA and chromosome CNA in cluster A. In 7 of these 10 genes, gene function was relevant to the direction of CNA, i.e., gain and loss of proto-oncogene and tumor suppressor genes, respectively. These 7 genes included the proto-oncogene, $L M O 3$, which reportedly plays a role in T-cell leukemia and brain tumors. The other 3 genes, which included PIK3R5, showed a loss of gene and chromosome copy number. Notably, this loss is functionally opposite because the genes are proto-oncogenic. These genes may be passenger genes and thus only useful as a lineage marker for the differentiation of clusters A and B.

Of the 93 genes that were reported to be important in a next-generation sequencing (NGS) analysis of breast cancers [29], 44 genes showed statistically significant differ-

Table 3. Comparisons of clinicopathological features and chromosomal changes between N0 and N1 samples of 57 invasive ductal carcinomas

\begin{tabular}{lrrl}
\hline & N0 & N1 & $p$ value \\
\hline Low NG $(1)$ & 9 & 3 & 0.5098 \\
High NG $(2,3)$ & 28 & 17 & \\
ER+ (luminal A/B; HER2+/-) & 31 & 12 & 0.0594 \\
ER- (HER2+/TN) & 6 & 8 & \\
$4 \mathrm{p}-$ & 7 & 4 & 1.0000 \\
$\quad$ Absent & 30 & 16 & \\
5p+ & 3 & 3 & 0.6542 \\
$\quad$ Absent & 34 & 17 & \\
6q- & 4 & 6 & 0.1410 \\
$\quad$ Absent & 33 & 14 & \\
14q- & 5 & 3 & 1.0000 \\
$\quad$ Absent & 32 & 17 & \\
16q- & 17 & 2 & 0.0077 \\
$\quad$ Absent & 20 & 18 & \\
17p- & 8 & 6 & 0.7651 \\
$\quad$ Absent & 29 & 18 & \\
22q- & 10 & 6 & 1.0000 \\
$\quad$ Absent & 27 & 14 & \\
\hline
\end{tabular}

NG, nuclear grade; TN, triple negative.

Progression Potential of DCIS Assessed by Genomic Copy Number Profiling ences in the mean CNA between clusters A and B (online suppl. file $3 \mathrm{~b}$ ), and 9 genes (GATA3, TP53, TET2, NCOR1, NOTCH2, PIC3CA, CREBBP, MYC, and ERBB2 in decreasing order of significance) showed concordance between both the gene and chromosome CNAs and the function and gain/loss of genes. Of these genes, only GATA3 and TP53 remained significant after Bonferroni correction. GATA3 gain and TP53 loss were common in cluster A.

\section{Discussion}

For validation of array CGH data, we utilized the samples with and without the overexpression $(3+)$ of HER2 protein and demonstrated almost complete concordance between array CGH and FISH results. In the absence of gene amplification, quantitative polymerase chain reaction as well as FISH is difficult to use for the validation of CNAs [20]. We attempted to cancel the noise inherent to formalin-fixed, paraffin-embedded tissues by averaging the $T / R$ ratio of larger-sized genes. Using the internal standard mentioned in the Methods, we optimized the gene size for the assessment of gene level copy number alterations. Applying this method to the present breast cancer samples, we found 2,828 genes of $\geq 4$ probes as the optimal condition for unsupervised clustering.

NGS showed that gene copy number changes are more common than significant DNA sequence changes in can-

Table 4. Comparisons of breast carcinomas between lineages A and $\mathrm{B}$

\begin{tabular}{lccl}
\hline & $\begin{array}{c}\text { A } \\
(n=50)\end{array}$ & $\begin{array}{l}\mathrm{B} \\
(n=31)\end{array}$ & $\begin{array}{l}p \\
\text { value }\end{array}$ \\
\hline Mean gain regions & 8.7 & 1.9 & 0.0023 \\
Mean loss regions $^{1}$ & 6.2 & 2.2 & 0.0143 \\
DCIS $^{1}$ & 10 & 14 & 0.0239 \\
IDC $^{1}$ & 40 & 17 & \\
${\text { Low NG }(1)^{1}}_{\text {High NG }(2,3)^{1}}$ & 7 & 15 & 0.0016 \\
ER+ (luminal A/B; HER2+/- $)^{1}$ & 43 & 16 & \\
ER- (HER2+/TN $)^{1}$ & 34 & 29 & 0.0117 \\
Mean Ki-67 index of cancer cells, $\%$ & 16 & 2 & \\
N+ in IDC cases $(n=30)$ & 6 & 3 & 1.6 \\
N- & 15 & 6 & \\
N+ in IDC samples $(n=57)^{1}$ & 14 & 6 & 1.00000 \\
N-1 & 26 & 11 & \\
\hline
\end{tabular}

NG, nuclear grade; TN, triple negative. ${ }^{1}$ Described in sample numbers. 


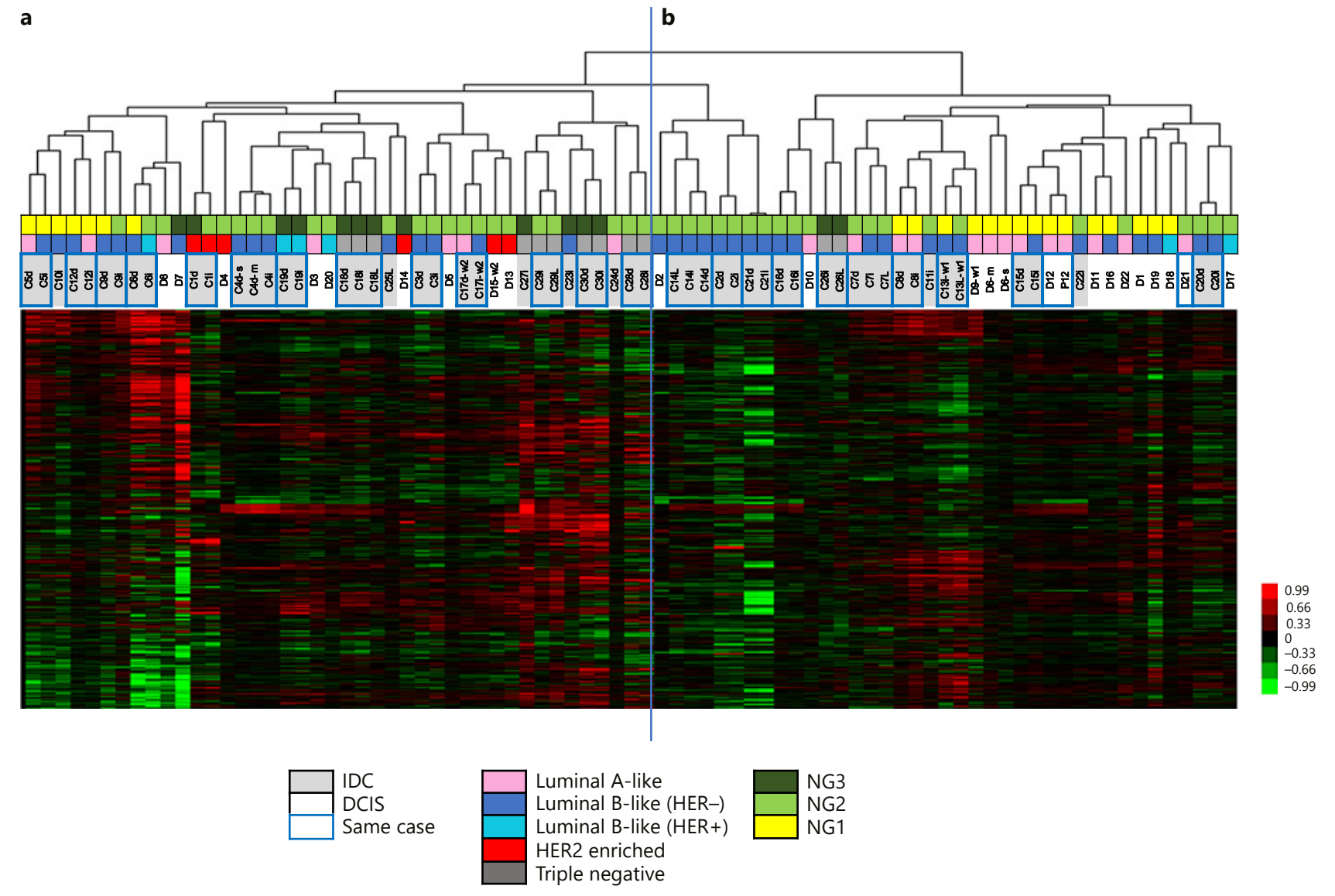

Fig. 2. a, b Unsupervised, hierarchical clustering using 81 samples and 2,828 larger genes that contain $\geq 4$ probes. The abbreviations of samples, D, Ci, Cd and CL indicate ductal carcinoma in situ (DCIS), ductal part of invasive ductal carcinoma (IDC), invasive part of IDC and lymph node metastasis, respectively. Copy number gains and losses are indicated by green and red squares, respec- tively, in the heat map. Beneath the dendrogram are 3 color bars. The top, the middle, and the bottom indicate nuclear grade (dark green, NG3; light green, NG2; yellow, NG1), intrinsic subtype (red, HER2; pink, luminal A; light and dark blue, luminal B; gray, triple negative), and tumor stage (white, DCIS; gray, IDC), respectively. Blue squares indicate sample groups of the same case).

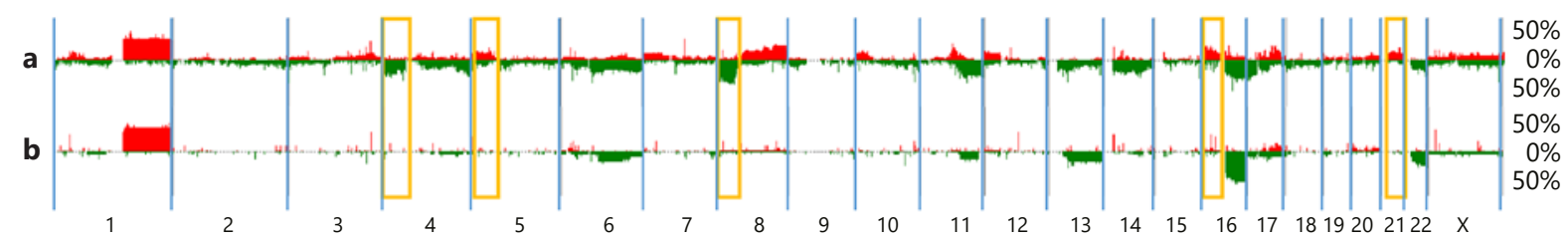

Fig. 3. Cluster-specific penetrance plots of ductal carcinomas. a Cluster A (50 samples). b Cluster B ( 31 samples). Yellow squares mark statistically significant changes. 
cers [29]. Additionally, recent NGS-based approaches to tumor heterogeneity demonstrated that chromosomal CNAs are the principal factor for tumor progression, whereas sequence changes of driver genes as well as chromosomal CNAs are important for the earlier phases of carcinogenesis [30]. In the present chromosome level CNA analysis, our penetrance plot of IDC (invasive part) showed a gain/loss pattern very similar to recent NGS data of 560 breast cancers [29], except for 9q loss, which was observed less frequently in our study.

The chromosomal CNA profiles were similar between DCIS and IDC, as previously reported [15, 31], except for the scarcity of losses in 6q, 11q, and 22q in DCIS (Table 1). Gains of $5 p, 7,11 q, 16 p$, and $20 q$ and a loss of $8 p$ were previously reported to be more frequent in IDC than DCIS $[32,33]$. In the present study, however, none of them showed a statistically significant difference between DCIS and IDC. Other clinicopathological factors were correlated with characteristic chromosomal changes (Tables 2, 3): 22q- correlated with high NG; $7 \mathrm{p}+, 8 \mathrm{q}+$, and $10 \mathrm{p}+$ with non-luminal-like (ER-) subtypes; the absence of $16 \mathrm{q}-$ with lymph node metastasis. The $8 \mathrm{q}+$, $17 q+$, and $8 p-$ reported to be common in high-grade breast cancers [31] were not significant in our results. CNAs, including a gain of $1 \mathrm{q}$ and loss of $16 \mathrm{q}$, were detected in nearly $50 \%$ of IDCs and $25 \%$ of DCISs. All samples with both $1 \mathrm{p}+$ and $16 \mathrm{q}-$ were of the luminal-like $(\mathrm{ER}+)$ type, as previously reported [34]. A problem is whether these changes are useful for the specification of progression-prone DCIS. Table 1 demonstrates that neither NG nor intrinsic subtype showed a significant correlation with an IDC/DCIS ratio, which may be related to the risk of progression from DCIS to IDC. Thus, we analyzed the following gene level CNAs to assess the outcome of individual tumors.

Under the above-mentioned optimal condition, the unsupervised hierarchical clustering gave 2 main clusters for all (cancer and papilloma) samples: A and B. The cancer samples of cluster A showed a greater frequency of chromosomal gain and loss, a greater IDC/DCIS ratio, a higher non-luminal-like/luminal-like (ER-/ER+) ratio and higher NG than cluster B (Table 4). Thus, the tumors of cluster A may be phenotypically less differentiated and have accumulated a greater number of genomic changes (Fig. 2, 3), reflecting a higher level of genomic instability. The DCIS lesions in cluster A that correlates with high NG may be more progression-prone to IDC than those in cluster B. This is consistent with the previous notion that NG largely paralleled the total number of gene CNAs and the risk of progression from DCIS to IDC $[31,35]$.

Progression Potential of DCIS Assessed by Genomic Copy Number Profiling
However, there was no difference in the frequency of lymph node metastasis between clusters A and B (Table 4) as well as between low and high NG and between ER+ and ER - tumors (Table 3). Additionally, Table 3 demonstrated that the accumulation of chromosomal CNAs was scarcely different between N0 and N1 samples. The only chromosomal CNA significantly different between N0 and $\mathrm{N} 1$ was the absence of $16 \mathrm{q}$ loss in metastasis samples. This suggests that the metastasis risk might be determined in an earlier phase of tumor development because the $16 \mathrm{q}$ loss is a common early event in breast carcinomas (Fig. 1). This point, whether the 16q copy number is useful in the prediction of metastasis risk, should be further studied using larger breast cancer cohorts.

It seems that the metastasis risk and the progression risk from DCIS to IDC reflect different genomic and epigenomic features. In stomach cancers, we similarly found that the copy number profiling approach could stratify tumor samples into 2 rapidly and slowly progressive groups, but no difference in metastasis risk was shown between these groups, whereas, different from the present study, metastatic gastric samples accumulated later chromosomal CNAs more frequently than the samples without metastasis (unpubl. data).

Of the 30,471 gene regions, 728 showed significant copy number differences between clusters $\mathrm{A}$ and $\mathrm{B}$. Among them, 9 genes (TP53, GATA3, CDKN2A, ATR, ATRX, PHF6, SMARCA4, APC, and ASXL1) were included in the 93 breast-cancer-related genes [29]. Of these 9 genes, only TP53 and GATA3 [36] remained significant after the Bonferroni correction and showed concordance between gene and chromosome changes and between gain/loss and gene function. The other genes showed discordance between gene function and copy number changes, and thus may be passenger genes (online suppl. file 3b). Recent meta-analysis of 10 articles reported that GATA3 expression was associated with better prognosis [37]. This seems contradictory to our result that invasionprone tumors often showed GATA3 copy number gain. In our unpublished data, copy number changes of GATA3 were not in parallel with its IHC results, which may reflect epigenetic regulations.

Still currently, screening-positive patients are often treated with additional radiotherapy after segmental surgical excision. Such postoperative therapies can be individualized based on the progression risk of each DCIS; the targets can be pinpointed to invasion-prone DCIS, as detected in the present study. The genes we specified could be useful for a construction of a simple system for pinpointing the invasion-prone DCIS. 


\section{Acknowledgment}

The present study was supported in part by JSPS KAKENHI Grant No. JP16K08689.

\section{Disclosure Statement}

The authors have no conflicts of interest to declare.

\section{References}

1 Kurebayashi J, Miyoshi Y, Ishikawa T, Saji S, Sugie T, Suzuki T, et al. Clinicopathological characteristics of breast cancer and trends in the management of breast cancer patients in Japan: Based on the Breast Cancer Registry of the Japanese Breast Cancer Society between 2004 and 2011. Breast Cancer. 2015 May;22(3):235-44.

2 Cowell CF, Weigelt B, Sakr RA, Ng CK, Hicks J, King TA, et al. Progression from ductal carcinoma in situ to invasive breast cancer: revisited. Mol Oncol. 2013 Oct; 7(5): 859-69.

3 Welch HG, Black WC. Using autopsy series to estimate the disease "reservoir" for ductal carcinoma in situ of the breast: how much more breast cancer can we find? Ann Intern Med. 1997 Dec;127(11):1023-8.

4 Independent UK Panel on Breast Cancer Screening. The benefits and harms of breast cancer screening: an independent review. Lancet. 2012 Nov;380(9855):1778-86.

5 Welch HG, Black WC. Overdiagnosis in cancer. J Natl Cancer Inst. 2010 May;102(9): 605-13.

6 Sagara Y, Mallory MA, Wong S, Aydogan F, DeSantis S, Barry WT, et al. Survival Benefit of Breast Surgery for Low-Grade Ductal Carcinoma In Situ: A Population-Based Cohort Study. JAMA Surg. 2015 Aug;150(8):739-45.

7 Page DL, Dupont WD, Rogers LW, Jensen RA, Schuyler PA. Continued local recurrence of carcinoma 15-25 years after a diagnosis of low grade ductal carcinoma in situ of the breast treated only by biopsy. Cancer. 1995 Oct;76(7):1197-200.

8 Stuart KE, Houssami N, Taylor R, Hayen A, Boyages J. Long-term outcomes of ductal carcinoma in situ of the breast: a systematic review, meta-analysis and meta-regression analysis. BMC Cancer. 2015 Nov;15(1):890.

9 Leonard GD, Swain SM. Ductal carcinoma in situ, complexities and challenges. J Natl Cancer Inst. 2004 Jun;96(12):906-20.

10 Amari M, Moriya T, Ishida T, Harada Y, Ohnuki K, Takeda M, et al. Loss of heterozygosity analyses of asynchronous lesions of ductal carcinoma in situ and invasive ductal carcinoma of the human breast. Jpn J Clin Oncol. 2003 Nov;33(11):556-62.

11 Yen MF, Tabár L, Vitak B, Smith RA, Chen HH, Duffy SW. Quantifying the potential problem of overdiagnosis of ductal carcinoma in situ in breast cancer screening. Eur J Cancer. 2003 Aug;39(12):1746-54.
12 Robanus-Maandag EC, Bosch CA, Kristel PM, Hart AA, Faneyte IF, Nederlof PM, et al. Association of C-MYC amplification with progression from the in situ to the invasive stage in C-MYC-amplified breast carcinomas. J Pathol. 2003 Sep;201(1):75-82.

13 Jang M, Kim E, Choi Y, Lee H, Kim Y, Kim J, et al. FGFR1 is amplified during the progression of in situ to invasive breast carcinoma. Breast Cancer Res. 2012 Aug; 14(4):R115.

14 Burkhardt L, Grob TJ, Hermann I, Burandt E, Choschzick M, Jänicke F, et al. Gene amplification in ductal carcinoma in situ of the breast. Breast Cancer Res Treat. 2010 Oct; 123(3):757-65.

15 Hernandez L, Wilkerson PM, Lambros MB, Campion-Flora A, Rodrigues DN, Gauthier A, et al. Genomic and mutational profiling of ductal carcinomas in situ and matched adjacent invasive breast cancers reveals intra-tumour genetic heterogeneity and clonal selection. J Pathol. 2012 May;227(1):42-52.

16 Vincent-Salomon A, Lucchesi C, Gruel N, Raynal V, Pierron G, Goudefroye R, et al.; breast cancer study group of the Institut $\mathrm{Cu}$ rie. Integrated genomic and transcriptomic analysis of ductal carcinoma in situ of the breast. Clin Cancer Res. 2008 Apr;14(7): 1956-65.

17 Liao S, Desouki MM, Gaile DP, Shepherd L, Nowak NJ, Conroy J, et al. Differential copy number aberrations in novel candidate genes associated with progression from in situ to invasive ductal carcinoma of the breast. Genes Chromosomes Cancer. 2012 Dec; 51(12):1067-78

18 Friberg S, Mattson S. On the growth rates of human malignant tumors: implications for medical decision making. J Surg Oncol. 1997 Aug;65(4):284-97.

19 Sonoda A, Mukaisho K, Nakayama T, Diem VT, Hattori T, Andoh A, et al. Genetic lineages of undifferentiated-type gastric carcinomas analysed by unsupervised clustering of genomic DNA microarray data. BMC Med Genomics. 2013 Jul;6(1):25.

20 Vo DT, Nakayama T, Yamamoto H, Mukaisho K, Hattori T, Sugihara H. Progression risk assessments of individual non-invasive gastric neoplasms by genomic copy-number profile and mucin phenotype. BMC Med Genomics. $2015 \mathrm{Feb} ; 8(1): 6$.
21 Furuya C, Kawano H, Yamanouchi T, Oga A, Ueda J, Takahashi M. Combined evaluation of CK5/6, ER, p63, and MUC3 for distinguishing breast intraductal papilloma from ductal carcinoma in situ. Pathol Int. 2012 Jun;62(6):381-90.

22 Wolff AC, Hammond ME, Hicks DG, Dowsett M, McShane LM, Allison KH, et al.; American Society of Clinical Oncology; College of American Pathologists. Recommendations for human epidermal growth factor receptor 2 testing in breast cancer: American Society of Clinical Oncology/College of American Pathologists clinical practice guideline update. Arch Pathol Lab Med. 2014 Feb;138(2):241-56.

23 Little SE, Vuononvirta R, Reis-Filho JS, Natrajan R, Iravani M, Fenwick K, et al. Array $\mathrm{CGH}$ using whole genome amplification of fresh-frozen and formalin-fixed, paraffinembedded tumor DNA. Genomics. 2006 Feb;87(2):298-306.

24 Barrett MT, Scheffer A, Ben-Dor A, Sampas $\mathrm{N}$, Lipson D, Kincaid R, et al. Comparative genomic hybridization using oligonucleotide microarrays and total genomic DNA. Proc Natl Acad Sci USA. 2004 Dec;101(51): 17765-70.

25 Eisen MB, Spellman PT, Brown PO, Botstein D. Cluster analysis and display of genomewide expression patterns. Proc Natl Acad Sci USA. 1998 Dec;95(25):14863-8.

26 Quackenbush J. Computational analysis of microarray data. Nat Rev Genet. 2001 Jun; 2(6):418-27.

27 Goldhirsch A, Winer EP, Coates AS, Gelber $\mathrm{RD}$, Piccart-Gebhart $M$, Thürlimann $B$, et al.; Panel members. Personalizing the treatment of women with early breast cancer: highlights of the St Gallen International Expert Consensus on the Primary Therapy of Early Breast Cancer 2013. Ann Oncol. 2013 Sep;24(9):2206-23.

28 Prat A, Cheang MC, Martín M, Parker JS, Carrasco E, Caballero R, et al. Prognostic significance of progesterone receptor-positive tumor cells within immunohistochemically defined luminal A breast cancer. J Clin Oncol. 2013 Jan;31(2):203-9.

29 Nik-Zainal S, Davies H, Staaf J, Ramakrishna M, Glodzik D, Zou X, et al. Landscape of somatic mutations in 560 breast cancer whole-genome sequences. Nature. 2016 Jun; 534(7605):47-54. 
30 Uchi R, Takahashi Y, Niida A, Shimamura T, Hirata H, Sugimachi K, et al. Integrated multiregional analysis proposing a new model of colorectal cancer evolution. PLoS Genet. 2016 Feb;12(2):e1005778.

31 Lopez-Garcia MA, Geyer FC, Lacroix-Triki M, Marchió C, Reis-Filho JS. Breast cancer precursors revisited: molecular features and progression pathways. Histopathology. 2010 Aug;57(2):171-92.

32 Yao J, Weremowicz S, Feng B, Gentleman RC, Marks JR, Gelman R, et al. Combined cDNA array comparative genomic hybridization and serial analysis of gene expression analysis of breast tumor progression. Cancer Res. 2006 Apr;66(8):4065-78.
33 Anbazhagan R, Fujii H, Gabrielson E. Allelic loss of chromosomal arm $8 \mathrm{p}$ in breast cancer progression. Am J Pathol. 1998 Mar;152(3): 815-9.

34 Buerger H, Otterbach F, Simon R, Schäfer KL, Poremba C, Diallo R, et al. Different genetic pathways in the evolution of invasive breast cancer are associated with distinct morphological subtypes. J Pathol. 1999 Dec; 189(4):521-6.
35 Fidalgo F, Rodrigues TC, Pinilla M, Silva AG, Maciel MS, Rosenberg C, et al. Lymphovascular invasion and histologic grade are associated with specific genomic profiles in invasive carcinomas of the breast. Tumour Biol. 2015 Mar;36(3):1835-48.

36 Takaku M, Grimm SA, Wade PA. GATA3 in Breast Cancer: Tumor Suppressor or Oncogene? Gene Expr. 2015;16(4):163-8.

37 Guo Y, Yu P, Liu Z, Maimaiti Y, Chen C, Zhang Y, et al. Prognostic and clinicopathological value of GATA binding protein 3 in breast cancer: A systematic review and metaanalysis.PLoSOne.2017 Apr;12(4):e0174843. 